\title{
Colonic Endometriosis: Dig Deeper for Diagnosis
}

\author{
Kanthi Rekha Badipatla ${ }^{\mathrm{a}, \mathrm{b}, \mathrm{d}}$, Anisha Vupputuria ${ }^{\mathrm{a}}$, Masooma Niazic, \\ Marie-Nirva Blaise ${ }^{a, b}$, Suresh Kumar Nayudu ${ }^{\text {a, b }}$
}

\begin{abstract}
Endometriosis is a common gynecological condition wherein there is an ectopic implantation of the uterine endometrial tissue. While several diagnostic modalities are described for the condition, laparoscopy remains the gold standard. There is still an undiscovered area to diagnose colonic endometriosis at an earlier stage. We present a case report of a reproductive age woman with cyclical rectal bleeding diagnosed with colonic endometriosis with colonoscopy and biopsy using saline injection lift and sampling technique. We in our report try to impress the fact that this differential should always be considered in the appropriate clinical setting, especially in women of childbearing age and in such cases, deeper tissue sampling techniques should be sought for, given better diagnostic yield. This may be clinically important given that it may aid in earlier diagnosis and thereby early initiation of appropriate therapy before the disease takes a complicated route. It may also be helpful in avoiding unnecessary surgery, along with the morbidity, complications and costs associated with same.
\end{abstract}

Keywords: Rectal bleeding; Colonic endometriosis

\section{Introduction}

Endometriosis is a disease in which endometrial epithelium is implanted outside the uterus. Estimated prevalence is about $10-15 \%$ in women of reproductive age group [1]. Gastrointestinal involvement is known to occur in $3-37 \%$ of the cases with recto-sigmoid being the most common site of involvement

Manuscript accepted for publication January 09, 2017

aDepartment of Medicine, Bronx Lebanon Hospital Center, Affiliated with Icahn School of Medicine at Mount Sinai, Bronx, NY, USA

${ }^{b}$ Division of Gastroenterology, Bronx Lebanon Hospital Center, Affiliated with Icahn School of Medicine at Mount Sinai, Bronx, NY, USA

'Department of Pathology, Bronx Lebanon Hospital Center, Affiliated with Icahn School of Medicine at Mount Sinai, Bronx, NY, USA

${ }^{\mathrm{d}}$ Corresponding Author: Kanthi Rekha Badipatla, Department of Medicine, Bronx Lebanon Hospital Center, Affiliated with Icahn School of Medicine at Mount Sinai, 1770 Grand Concourse, Apt \#6B, Bronx, NY 10457, USA. Email:kanthirb@gmail.com

doi: https://doi.org/10.14740/gr760e
[2]. Prompt diagnosis involves integration of clinical manifestations, imaging techniques, biomarkers and surgical techniques. Laparoscopy which is considered as the gold standard in endometriosis diagnosis has considerable procedure-related risks [3]. While colonoscopy with biopsy is traditionally not considered a diagnostic tool for colorectal endometriosis [4], we open doors to a new diagnostic option of deeper tissue sampling and avoid surgery and the associated morbidity and mortality.

\section{Case Report}

A 43-year-old female patient presented to the gastroenterology clinic with complaints of rectal bleeding and constipation. She reported episodes of bloody stools cyclically coinciding with her menstrual periods with burning rectal pain, and she also reported long standing constipation for many years for which she has been taking laxatives. She also had lower abdominal pain, colicky in nature, improved with mucoid bowel movement, and associated with a sensation of incomplete evacuation and abdominal bloating. She denied fever, diarrhea, joint pains or skin rash. She denied any nausea, vomiting, loss of appetite and weight loss at the time of initial presentation.

Her medical history was negative for any chronic medical conditions. She did not undergo any surgical procedures in the past. There were no gastrointestinal malignancies diagnosed in her immediate or distant family members. She never used tobacco products, alcohol or recreational drugs. She was not allergic to any medications.

On initial evaluation, her vital signs were within normal limits. Abdomen was non-distended, soft and non-tender to palpation. On auscultation, bowel sounds were noted to be normoactive. Rectal examination was unremarkable. Cardiorespiratory and neurological examination was within normal limits.

She underwent flexible colonoscopy under monitored anesthesia care. Examination revealed an $8-10 \mathrm{~cm}$ area of erythematous, congested and granular mucosa in the sigmoid colon (Fig. 1). Random biopsies done showed colonic mucosa with focal erosion and mild chronic inflammation. The area was tattooed for future reference. Also, noted during colonoscopy was non-bleeding hemorrhoid. In the interim patient continued to be symptomatic.

While pursuing surgical consultation for the management of hemorrhoids, we decided to repeat a flexible colonoscopy 

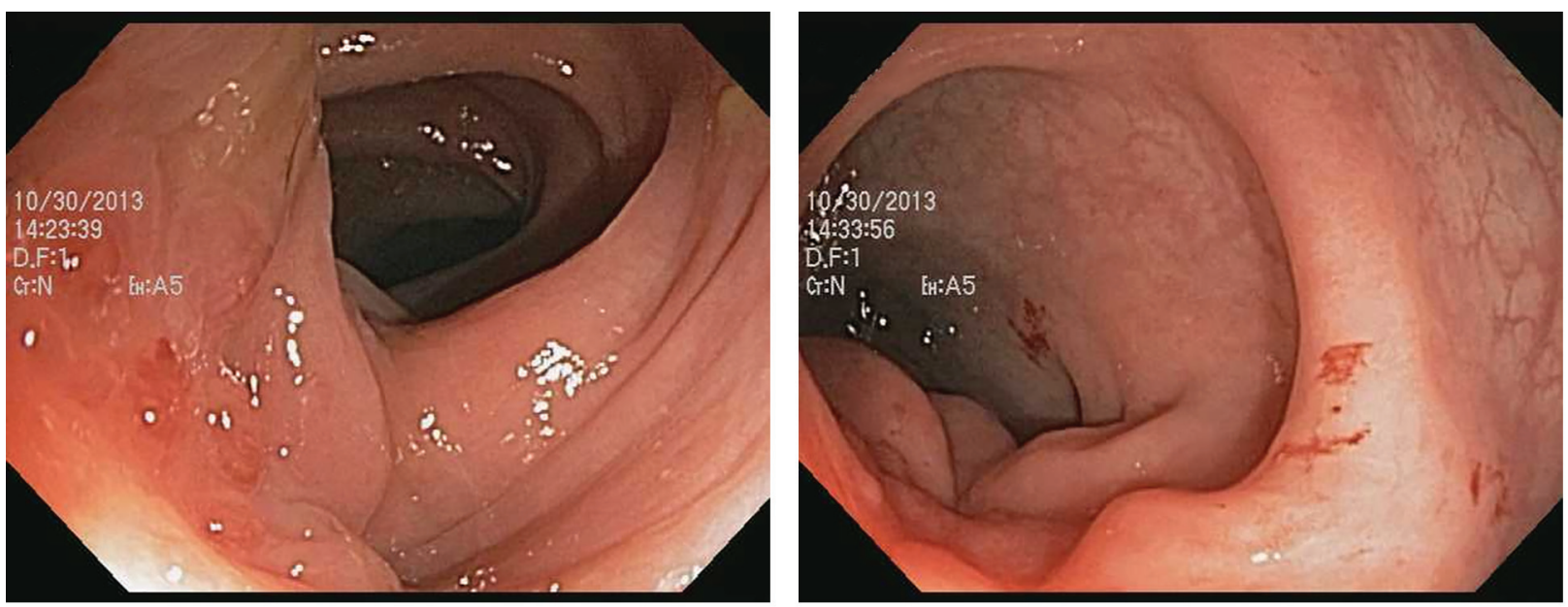

Figure 1. Colonoscopy image of abnormal polypoid granular mucosa.

with tissue sampling in view of her cyclical symptoms and initial colonoscopy findings. On repeat examination, the area was injected and raised using $5 \mathrm{~mL}$ of saline to create a fluid bed that separated the tissue layers from underlying muscle layer. Deeper tissue samples were obtained using cold forceps. Histopathological examination revealed fragments of mucosa consistent with endometriosis (Fig. 2). Immunohistochemical staining was positive for CK-7 and CD10 (Fig. 3) which were consistent with the diagnosis of endometriosis. Interestingly further enquiry and review of her past record revealed her prior history of endometriosis. She was referred for a gynecological evaluation and successfully underwent elective hysterectomy with bilateral salpingo-oophorectomy. On follow-up visits, she reported complete resolution of symptoms.

\section{Discussion}

Endometriosis is a hormone responsive pathological condition characterized by ectopic implantation of uterine endometrial tissue. Colorectal involvement can happen as a part of the disease spectrum. Symptoms include alteration in bowel habits, dyschezia, and rectal bleeding [5], all of which when happening in a reproductive age group female and cyclical manner should prompt the clinician to consider endometriosis as a part differential diagnosis. Complications including bowel obstruction, stricture and perforation may occur [6]. Volvulus [7] and intussusception [8] secondary to bowel involvement have also been described in the literature.

Common clinical conditions which mask or mimic colonic endometriosis include irritable bowel syndrome, inflammatory bowel disease [9] and malignancy [10]. Review of literature also shows that there are reported cases wherein when malignancy was considered high in the differential, surgical management was pursued [4]. While macroscopically identifiable lesions are found in a subset of patients with bowel endometriosis, some patients may also have occult microscopic involvement as well [11].

Good clinical history and correlation of symptoms in appropriate clinical setting are key in suspecting the diagnosis. This can be aided by appropriate radiological assessment [12]. Endo-sonography may reveal pathology in the deeper layers [13]. However, histopathological diagnosis remains confirmatory. This is true especially in patients who do not carry a
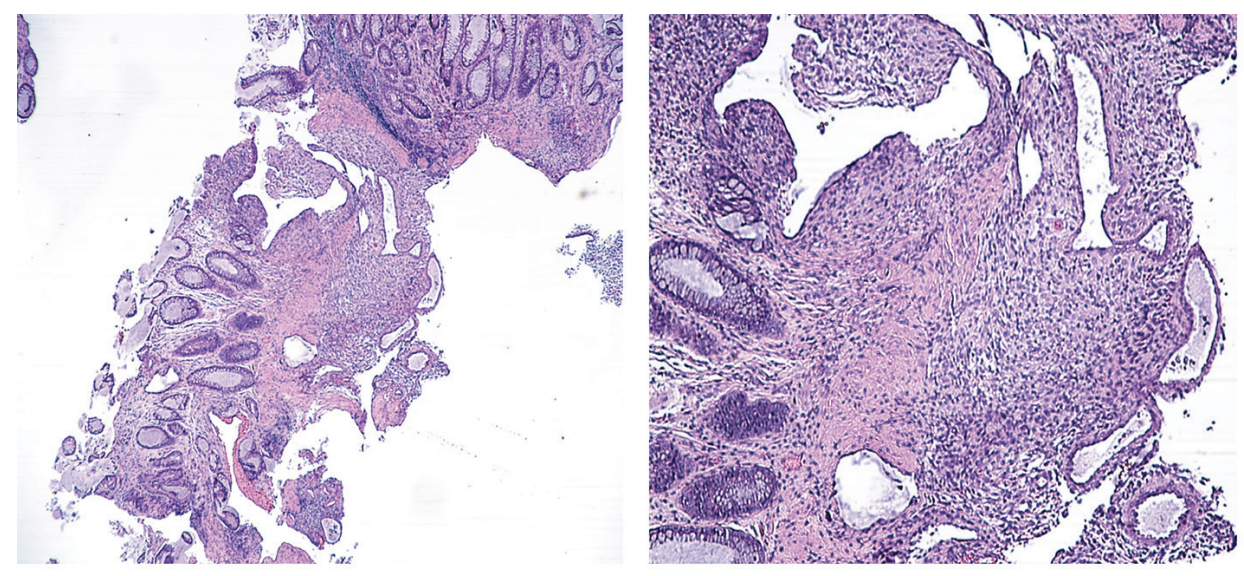

Figure 2. Colonic mucosa with endometriosis. 

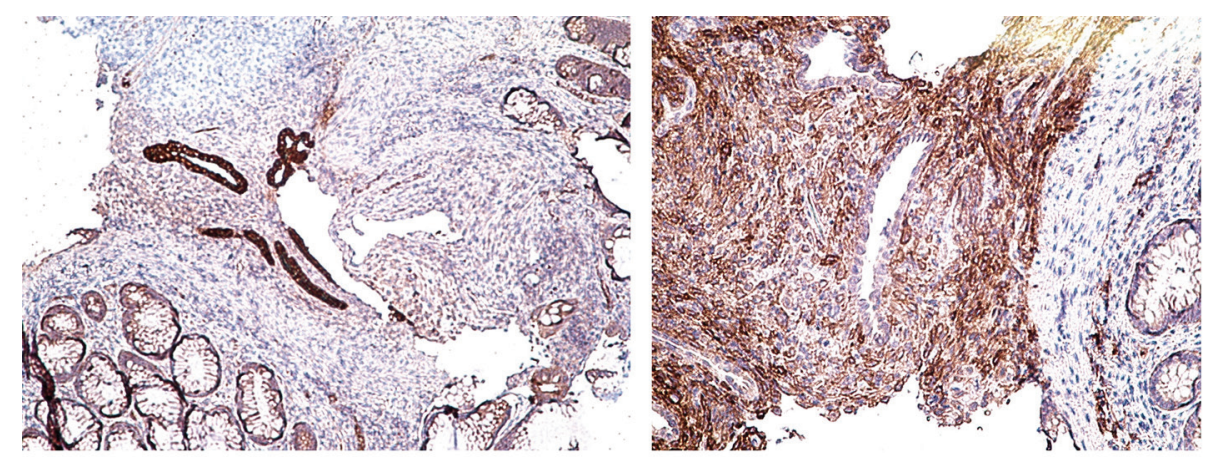

Figure 3. Immunostaining positive for CK-7 and CD10.

preliminary diagnosis of pelvic endometriosis. Colonic lesions when encountered in such patients leave the endoscopist with a broader differential diagnosis which include malignancy and thus prompting them to pursue surgical management.

Generally, endometriosis is managed by either hormonal or surgical therapies, the latter being recommended for women who are above 40 years of age and do not want to retain their reproductive function [14]. Hormonal therapy for colonic endometriosis can be a potential option given the hormone responsive nature of the condition and tropism of the endometrial tissue to estrogen in uncomplicated patients who receive early diagnosis [15]; However, long-term effects of such treatment are unknown. This may be limited by usual delays in diagnosis. Our patient chose to have a salpingo-oophorectomy done with which she had good symptom resolution. Colonic stenting can be an option in patients who present with obstruction as a bridge to surgery [16]. Surgery is always the option in complicated cases presenting with acute abdomen and unresponsive cases with extensive disease.

There exist no current guidelines on colonoscopy in cases of pelvic endometriosis and study conducted by Milone et al did not justify routine colonoscopy in known cases of pelvic endometriosis given the diagnostic low yield [17]. There are also no specific guidelines for surveillance in treated cases. There is currently no existing data in support of post-operative hormonal therapy given the possibility of occult microscopic involvement. Probably more future studies are needed in these areas to further guide clinicians in managing endometriosis involving colon.

Diagnostic delay of endometriosis is still an area of concern. The best method for early diagnosis of colorectal endometriosis is an area to be explored further. We recommend that deeper tissue sampling techniques wherever possible will help to achieve diagnosis less invasively. Also, endoscopic tissue sampling may avoid surgical interventions, thereby limiting extensive bowel surgeries and associated complications. We end by saying that "endoscopist to dig deeper" for bowel endometriosis in the right setting.

\section{Conflicts of Interest}

None.

\section{References}

1. Riazi H, Tehranian N, Ziaei S, Mohammadi E, Hajizadeh E, Montazeri A. Clinical diagnosis of pelvic endometriosis: a scoping review. BMC Womens Health. 2015;15:39.

2. Montalto M, Santoro L, D'Onofrio F, Gallo A, Campo S, Campo V, Gasbarrini A, et al. Endometriosis, need for a multidisciplinary clinical setting: the internist's point of view. Intern Emerg Med. 2010;5(6):463-467.

3. Luisi S, Lazzeri L, Ciani V, Petraglia F. Endometriosis in Italy: from cost estimates to new medical treatment. Gynecol Endocrinol. 2009;25(11):734-740.

4. Rana R, Sharma S, Narula H, Madhok B. A case of rectosigmoid endometriosis mimicking carcinoma. Springerplus. 2016;5:643.

5. Rambuszek P, Milek T. Rectal endometriosis - rare case of intestinal obstruction. Case report. Pol Przegl Chir. 2013;85(4):219-222.

6. Wolthuis AM, Meuleman C, Tomassetti C, D'Hooghe T, de Buck van Overstraeten A, D'Hoore A. Bowel endometriosis: colorectal surgeon's perspective in a multidisciplinary surgical team. World J Gastroenterol. 2014;20(42):15616-15623.

7. Ito D, Kaneko S, Morita K, Seiichiro S, Teruya M, Kaminishi M. Cecal volvulus caused by endometriosis in a young woman. BMC Surg. 2015;15:77.

8. Khwaja SA, Zakaria R, Carneiro HA, Khwaja HA. Endometriosis: a rare cause of small bowel obstruction. BMJ Case Rep. 2012;2012.

9. Dong C, Ngu WS, Wakefield SE. Endometriosis masquerading as Crohn's disease in a patient with acute small bowel obstruction. BMJ Case Rep. 2015;2015.

10. Chen H, Luo Q, Liu S, Xiong H, Jiang Q. Rectal mucosal endometriosis primarily misinterpreted as adenocarcinoma: a case report and review of literature. Int J Clin Exp Pathol. 2015;8(5):5902-5907.

11. Badescu A, Roman H, Aziz M, Puscasiu L, Molnar C, Huet E, Sabourin JC, et al. Mapping of bowel occult microscopic endometriosis implants surrounding deep endometriosis nodules infiltrating the bowel. Fertil Steril. 2016;105(2):430-434 e426.

12. Rousset P, Peyron N, Charlot M, Chateau F, Golfier F, Raudrant D, Cotte E, et al. Bowel endometriosis: preop- 
erative diagnostic accuracy of 3.0-T MR enterography initial results. Radiology. 2014;273(1):117-124.

13. Lee TH, Lee JS, Lee DW, Kim JO. Isolated bowel endometriosis resembling a myogenic tumor on endoscopic ultrasonography. Korean J Intern Med. 2012;27(3):353355.

14. Moawad NS, Caplin A. Diagnosis, management, and long-term outcomes of rectovaginal endometriosis. Int $\mathrm{J}$ Womens Health. 2013;5:753-763.

15. Leone Roberti Maggiore U, Scala C, Remorgida V, Ven- turini PL, Del Deo F, Torella M, Colacurci N, et al. Triptorelin for the treatment of endometriosis. Expert Opin Pharmacother. 2014;15(8):1153-1179.

16. Whelton $\mathrm{C}$, Bhowmick A. Acute endometrial bowel obstruction-A rare indication for colonic stenting. Int J Surg Case Rep. 2013;4(2):160-163.

17. Milone M, Mollo A, Musella M, Maietta P, Sosa Fernandez LM, Shatalova O, Conforti A, et al. Role of colonoscopy in the diagnostic work-up of bowel endometriosis. World J Gastroenterol. 2015;21(16):4997-5001. 